\title{
Screening Contracts in the Presence of Positive Network Effects*
}

\author{
Gergely Csorba ${ }^{\dagger}$ \\ Central European University
}

March 24, 2004

\begin{abstract}
Based on the critical assumption of strategic complementarity, this paper builds a general model to describe and solve the screening problem faced by the monopolist seller of a network good. By applying monotone comparative static tools, we demonstrate that the joint presence of asymmetric information and positive network effects leads to a strict downward distortion for all consumers in the quantities provided. We also show that the equilibrium allocation is an increasing function of the intensity of network effects, and that a discriminating monopoly may supply larger quantities for all consumers than a competitive industry.
\end{abstract}

Keywords: network effects, strategic complementarities, contracting with externalities, second-degree discrimination, monotone comparative statics

JEL Classification: D42, D62, D82, L12

*The author is very much indepted to Peter Benczur for his detailed comments, and thanks to Attila Ambrus, Russell Cooper and Patrick Rey for useful discussions, as well as seminar participants at the 2003 Villa Mondragone Workhop in Rome and the 2003 Econometric Society European Meeting in Stockholm.

${ }^{\dagger}$ Postal address: Central European University, Economics Department, Nador u. 9, 1051 Budapest, Hungary. Phone: 361327 3020, Fax: 3613273232 . E-mail address: cphcsg01@phd.ceu.hu 


\section{Introduction}

This paper derives a general model of second-degree discrimination in the presence of positive network effects. Positive network effects are also called strategic complementarities in consumption, and are present if an economic agent's utility derived from the consumption of the good is positively affected by the consumption level (or number) of other agents consuming the same or compatible products. ${ }^{1}$ These effects commonly arise in various modern industries, like telecommunications, hardware and software or banking, and these industries are similar in the following properties: they are highly concentrated and the firms use a wide variety of nonlinear tariffs or very detailed contracts. Our goal in this paper is to use the tools of monotone comparative statics to describe the screening problem faced by a monopolist seller of a network good, and to give a complete characterization of the optimal contracts it can use.

We build a unifying framework to examine two well-known results, which are usually referred separately in the analysis of network economics. The first is one of the main conclusions in second-degree discrimination models, namely that the incentive problem due to information asymmetry makes the monopoly distort the quantity supplied to consumers with smaller willingness to pay for the good, but makes 'no distortion at the top': consumers with the largest willingness to pay are provided with the first-best optimal quantities. ${ }^{2}$ The second result is due to the externality literature: once a consumer's utility is not only the function of his own consumption level, but of the others' consumption levels as well, then in equilibrium all economic agents end up with socially suboptimal quantities. ${ }^{3}$ Network effects, which are generally assumed to be positive, result in underconsumption of the network good for all consumers.

\footnotetext{
${ }^{1}$ For the implications of strategic complementarities on the production side (positive production externalities) in principal-agent models, see Lockwood (2000).

${ }^{2}$ The seminal results of the second-degree discrimination literature were derived by Mussa and Rosen (1978) and Maskin and Riley (1984), summarized for example in Fudenberg and Tirole (1991) and Laffont and Martimort (2002).

${ }^{3}$ For a general overview on externalities, see for example Laffont (1988).
} 
The main aim of this paper is to show that if asymmetric information and positive network effects are both present, these two impacts reinforce each other, so there will be a strict downward distortion for all consumers in the quantities provided. We also find that despite the downward distorting impact of positive network effects, the equilibrium outcome is an increasing function of the intensity of the network effects, no matter which type of discrimination we consider. The first result is a theoretical contribution to the literature on optimal screening, and together with the second it has important implications for optimal pricing policies in network economies. Last, we show that in some cases the discriminating monopoly supplies larger quantities for all consumers than a perfectly competitive industry, which result may be relevant for regulatory economics in these industries.

Let us demonstrate the strict downward distortion result by a simple example. Suppose there are only two consumers of a network good, let us call them sophisticated and normal. Assume that the sophisticated consumer benefits more both from his individual consumption and from network size, where the latter is now identified as total consumption level. Whenever the monopoly is capable of perfectly discriminating between the two consumers, it grasps both consumers' surplus and supplies the welfare-maximizing quantities. However, when the monopoly is restricted to offer the same menu of contracts to both consumers, standard incentive theory tells us that it should distort the quantity devoted to the normal consumer downwards in order to make switching less attractive to the sophisticated consumer. Now if the normal consumer's quantity decreases, so does network size, and since positive network effects are present, the sophisticated consumer's utility from his individual consumption is negatively affected. Thus, it is no more feasible to offer him the first-best optimal quantity, and his consumption should be distorted downwards as well.

Before starting with the main model, we briefly discuss the related literature. The "old literature" on network effects, which was basically on telecommunication pricing, focused on the question whether the network size would be the 
one that maximizes social welfare. ${ }^{4}$ It was found that in perfect competition the network effects cannot be fully internalized, and a monopoly may perform better, since it controls both the price and the quantity, and may use crosssubsidization policies more effectively. ${ }^{5}$ Our analysis reinforces the result that the perfectly discriminating monopoly always supplies larger quantities for all consumers than a perfectly competitive industry, since it may set the socially optimal allocation and reap the increased surplus of each consumer. However, the comparison of second-best discrimination and perfect competition allocations does not give unambiguous results, since we are comparing two outcomes that fail to be the first-best for two different reasons: incentive problems due to information asymmetry and the incapability of internalizing network effects.

The "new literature" on network effects considered mainly homogeneous types of consumers and concentrated on the multiple equilibria problem created by different (rational) expectations. ${ }^{6}$ Models with heterogeneous types of consumers were only recently used by Fudenberg and Tirole (2000) and Ellison and Fudenberg (2001). However, these models analyze the effectiveness of dynamic strategies, like entry deterrence or software upgrades, in the presence of network effects, hence they concentrate on intertemporal discrimination aspects. Hahn (2003) builds a special model of telecommunication to examine the role of call and network externalities in nonlinear pricing. He establishes the result that in equilibrium all types end up with suboptimal quantities, so the "no distortion on the top' result does not hold. Nevertheless, since he works with a special utility structure, he attributes this result to the existence of call externalities.

Two works closely related to ours are Segal $(1999,2003)$, which develop a

\footnotetext{
${ }^{4}$ Seminal papers include Rohlfs (1974), Littlechild (1975) and Oren et al. (1982). This classification between the old and new literature on network effects is based on Liebowitz and Margolis (2002).

${ }^{5}$ Similar conclusions have been derived in the macroeconomics literature on imperfect competition, for example in Cooper and John (1988, p. 454): "a demand externality may arise, though, in market structures where agents require information on both prices and quantities in making choices [...] In these cases quantities matter to individual decision makers, and prices do not completely decentralize allocations".

${ }^{6}$ Farrell and Saloner (1985) and Katz and Shapiro (1985) were the first to raise this problem.
} 
general model of contracting with externalities and characterize the nature of the arising inefficiencies. When externalities are positive, Segal shows that each agent's consumption level is smaller in the resulting equilibrium allocation than in the socially efficient one. Strategic complementarity is identified as the factor accounting for this general feature $;^{7}$ however, the analysis names two additional assumptions that are useful in identifying the direction of distortions: first, the consumers are identical (hence there are no information asymmetries), and second, total welfare depends only on aggregate trade, and not on its allocation across consumers. ${ }^{8}$

This paper shows that the underconsumption result holds without these simplifying assumptions if externalities are positive. In our model consumers are heterogenous in two respects: first, they have different (exogenously given) valuations towards the same menus, and second, depending on their (endogenous) choices, they may have different valuations for the same network. ${ }^{9}$ This setting can be applied both to networks where the agents are screened by the different consumption or usage level (such as in telecommunication), and to networks where agents have unit demand for the good and are screened by the quality of the service (such as in software markets).

Strategic complementarity, which is an inherent characteristic of positive network effects, is the "critical assumption" in the terminology of Milgrom and Roberts (1994) that drives our results. It allows us to characterize the optimal contracts in a general setting by applying monotone comparative static tools, pioneered by Topkis (1978) and Milgrom and Shannon (1994). The main advantage of our approach is that instead of solving the model explicitly for the different first- and second-best allocations, it gives a simple method to compare the equilibrium allocations. We develop a parametrized functional form that encompasses both regime as optimal solutions for different parameter values,

\footnotetext{
${ }^{7}$ Segal (1999, p. 356) names this key property "increasing externality".

${ }^{8}$ Segal (1999, p. 341).

${ }^{9}$ The second property works for the other direction as well: different networks sizes result in different marginal utilities of the same individual consumption for different consumers.
} 
and then show that the optimal solution is a strictly monotone function of this parameter.

The rest of this paper is organized as follows. Section 2 introduces the main setup of the model, then Section 3 presents the benchmark case of first-best discrimination. In Section 4 we turn to the implementation of an incentivecompatible menu of contracts, and characterize the second-best optimal contract in the presence of positive network effects. Section 5 compares the outcomes of the two previous sections and analyzes further comparative statics questions. Since monotone comparative static tools can be more suitably used for sets of finite dimensions, the main model is built for a discrete distribution of types. In Section 6 we briefly discuss the continuous type case. Finally, Section 7 concludes and discusses the possible extensions of the model.

\section{The model}

Consider a monopoly that produces a good exhibiting positive network effects at a constant marginal cost $c$. We assume that the network goods sold to the consumers are perfectly compatible with each other. Consumers have heterogenous preferences for the good, a consumer of type $\theta_{i}$ is assumed to have a utility function of

$$
U\left(\theta_{i}\right)=\theta_{i} V\left(q_{i}, \bar{q}\right)-t_{i},
$$

where $q_{i}$ is the amount of the network good he consumes, $\bar{q}=\sum_{i} q\left(\theta_{i}\right) f\left(\theta_{i}\right)$ is the total amount of network good in the economy (network size), and $t_{i}$ is the tariff charged for $q_{i}$ by the monopoly.

Suppose there are $n$ different types of consumers, so that $\theta_{1}<\theta_{2}<\ldots<\theta_{n}$, and let $N$ denote the set of different types. Consumer types are independently distributed by a given cumulative distribution function $F(\theta)$, and this distribution function is common knowledge for all consumers and the monopoly. We assume that there is a continuum of consumers in each type, so a single consumer's contribution to the network is negligible. 
Suppose that there are no externalities on non-traders: for all $\bar{q}, V(0, \bar{q})=0$, so the outside option is zero for all consumers. ${ }^{10}$ However, we do not restrict our analysis to pure network goods, i.e. the stand-alone utility $V(q, 0)$ may differ from 0 . We assume that $V(\cdot)$ is twice continuously differentiable and that $V_{1}>0$ and $V_{11} \leq 0$, so the marginal utility of individual consumption level is positive and decreasing. ${ }^{11}$

The positivity of network effects is captured by the following two key assumptions. First, $V_{2}>0$, so the marginal utility of network size is always positive. Second, the consumption levels of each consumer groups are strategic complements, which is equivalent of stating that $V(\cdot)$ has increasing differences on $\left(q_{i}, q_{-i}\right){ }^{12}$ These two assumptions together imply that individual consumption and network size are strategic complements as well, thus $V_{12} \geq 0 .{ }^{13}$

Three remarks are in order. First, instead of the classical quantity discrimination approach, we could give another interpretation of this problem, where consumers have unit demands for the network good, and the goods differ in their quality $q_{i}$. If in this case we normalize the mass of consumers to $1, \bar{q}$ can be seen as average quality level in the network. Second, this functional form reflects the case as well, where each consumer looks at the usage levels of different packages as perfect substitutes in the network size, but gives a higher weight to the packages similar to his own. Formally, this would mean that $\bar{q}_{i}=\alpha q\left(\theta_{i}\right) f\left(\theta_{i}\right)+\beta \sum_{j \neq i} q\left(\theta_{j}\right) f\left(\theta_{j}\right)$, where $\alpha>\beta$. However, we can rewrite $\bar{q}_{i}$ as

\footnotetext{
${ }^{10}$ This property ensures that consumers' reservation value is type-independent. See Jullien (2000) for a general model presenting the complications arising from type-dependent reservation values.

${ }^{11}$ In all of this paper, lower indexes refer to partial derivatives of the function $V(\cdot)$ in its respective argument. Second, the words increasing (decreasing) and bigger (smaller) are used in the weak sense.

${ }^{12}$ A function $g(x, t)$ has increasing differences in $(x, t)$ if $g\left(x, t^{\prime}\right)-g(x, t)$ is increasing in $x$ for all $t^{\prime}>t$.

${ }^{13}$ Note that the strategic complementarity of group consumption levels is a stronger assumption, since it also captures that if a consumer's individual consumption level increases to $q_{i}^{\prime}$, the same will happen in equilibrium for all type- $i$ consumers, therefore network size changes as well (technically speaking, it is a total derivative instead of a partial one).
} 
$(\alpha-\beta) q\left(\theta_{i}\right) f\left(\theta_{i}\right)+\bar{q}$, where $\alpha-\beta>0$, and then an appropriate modification of the original $V(\cdot)$ function will capture this case as well, and satisfy the assumptions given above. Third, we have chosen constant marginal costs only for expositional simplicity. Our qualitative results remain unchanged if we allow for a cost function exhibiting weak cost complementarities (or so called economies of scope), a property that generally fits the structure of network industries. ${ }^{14}$

The timing of the model is the following. First, the monopoly offers a menu of contracts $\left\{\left(q_{i}, t_{i}\right)\right\}_{i=1}^{n}$, among which consumers will self-select. Second, consumers observe all possible contracts, and form their expectations about the network size $\bar{q}$. Finally, each consumer decides which package to purchase or buys nothing, and payoffs are made.

We require that consumers' expectations are rational, so they should be fulfilled in equilibrium. However, we should also define an equilibrium selection criterion, since as many models with network effects show, consumers' expectations about others' choices crucially influence their behavior, which may result in multiple equilibria. In our model the consumers' individual consumption levels are strategic complements, which induces a so-called supermodular game, so there will be a (rationally expected) equilibrium that Pareto-dominates the others. ${ }^{15}$ We assume that once the monopoly offers the menu of contracts $\{(q, t)\}$, consumers coordinate on this equilibrium. Milgrom and Roberts (1996) give a further justification to concentrate on the Pareto-dominant equilibrium by showing that it is the only coalition-proof (correlated) equilibrium under any admissible coalition communication structure.

\footnotetext{
${ }^{14}$ This is because weak cost complementarities imply a submodular cost function, as shown by Sharkey (1982). It is also a sufficient condition for the firm being a natural monopoly. ${ }^{15}$ See Theorem 7 in Milgrom and Roberts (1990).
} 


\section{The first-best optimal contract}

As a benchmark case, suppose that the monopoly knows each consumer's type, and based on this information it can offer personalized contracts to them. Then the contracts will be designed such that in equilibrium each type realizes nonnegative utility, that is

$$
\theta_{i} V\left(q_{i}, \bar{q}\right)-t_{i} \geq 0
$$

for all $i \in N$. Since the distribution function is common knowledge, each consumer rationally expects the network size $\bar{q}$ to be $\sum_{i \in N} q_{i} f\left(\theta_{i}\right)$.

The profit of the monopoly is given by

$$
\Pi=\sum_{i \in N}\left(t_{i}-c q_{i}\right) f\left(\theta_{i}\right)
$$

and it has to be maximized such that participation constraints $\left(P_{i}\right)$ are satisfied. Naturally, all constraints will be binding in optimum, so the optimal quantity schedule determines the optimal tariff schedule. Therefore, the key decision variable for the monopoly is $q=\left(q_{1}, \ldots, q_{n}\right) \in Q=R^{n}$, the latter set being a lattice. ${ }^{16}$ The function $V(\cdot)$ is defined on this lattice $Q$, and since we have assumed that any $q_{i}$ and $q_{j}(i \neq j)$ are strategic complements, it implies that $V(\cdot)$ is supermodular in $q$ on $Q .{ }^{17}$

The final form of the profit function is

$$
\Pi^{F B}=\sum_{i \in N} \theta_{i} V\left(q_{i}, \bar{q}\right) f\left(\theta_{i}\right)-c \bar{q} .
$$

Since there are no externalities on non-traders, the perfectly discriminating monopoly internalizes all network effects. Thus the monopoly's problem is equivalent to the welfare-maximizing one, and the optimal allocation produces

\footnotetext{
${ }^{16} \mathrm{~A}$ lattice is a partially ordered set, which contains the least upper bound (so called join) and greatest lower bound (so called meet) of each pair of its elements. The join (meet) of two elements $x^{\prime}$ and $x^{\prime \prime}$ is denoted by $x^{\prime} \vee x^{\prime \prime}\left(x^{\prime} \wedge x^{\prime \prime}\right)$. In $R^{2}, x^{\prime} \vee x^{\prime \prime}=\left(\max \left\{x_{1}^{\prime}, x_{1}^{\prime \prime}\right\}, \max \left\{x_{2}^{\prime}, x_{2}^{\prime \prime}\right\}\right)$ and $x^{\prime} \wedge x^{\prime \prime}=\left(\min \left\{x_{1}^{\prime}, x_{1}^{\prime \prime}\right\}, \min \left\{x_{2}^{\prime}, x_{2}^{\prime \prime}\right\}\right)$.

${ }^{17}$ This follows from Theorem 2.6.2. in Topkis (1998). A function $g(x)$ is supermodular on the lattice $X$, if $g\left(x^{\prime}\right)+g\left(x^{\prime \prime}\right) \leq g\left(x^{\prime} \vee x^{\prime \prime}\right)+g\left(x^{\prime} \wedge x^{\prime \prime}\right)$ for all $\left(x^{\prime}, x^{\prime \prime}\right) \in X$.
} 
no social inefficiency. This result is in line with Segal (1999), since so far the heterogeneity of consumers played no role.

Note that the profit function is the sum of supermodular functions multiplied by positive constants, so it will be supermodular on $Q .{ }^{18}$ In all of this paper, since we concentrate on the critical assumptions allowing to compare the first- and second best outcomes, we assume that the respective profit function has a positive bounded maximum, without imposing any sufficient conditions guaranteeing this property.

If we maximize $\Pi^{F B}$ in $q$, the following set of first-order conditions characterize the first-best allocation $q^{F B}: 19$

$$
\theta_{i} V_{1}\left(q_{i}, \bar{q}\right)+\sum_{j \in N} \theta_{j} V_{2}\left(q_{j}, \bar{q}\right) f\left(\theta_{j}\right)=c, \text { for all } i \in N .
$$

The first term measures the marginal utility of individual consumption for a consumer of type $i$, we will call it individual effect. The second term sums the marginal utility increases of all consumers due to the increased consumption of consumer group $i$, which will be called network effect. Note that the network effects are the same in all equations. If there are no network effects, we are back to the standard result of first-best implementation: individual effect should equal marginal cost.

By combining two first-order conditions, we have that

$$
\theta_{i} V_{1}\left(q_{i}, \bar{q}\right)=\theta_{j} V_{1}\left(q_{j}, \bar{q}\right)
$$

for all $i, j \in N$. Since $V_{11} \leq 0, \theta_{i}<\theta_{j}$ implies $q_{i}<q_{j}$, so in the first-best optimum consumers of higher types end up with a larger quantity level than consumers of lower types.

\footnotetext{
${ }^{18}$ See Lemma 2.6.1 in Topkis (1998). We use the fact that a one-dimensional function, like $c q_{i}$, is both super- and submodular, and a supermodular minus a submodular function is still supermodular.

${ }^{19}$ Throughout the whole paper, the final forms of the first-order conditions are derived after dividing the equations by the density of the respective type.
} 


\section{The second-best optimal contract}

If the monopoly should offer the same menu of contracts for all consumers, the first-best optimum is not feasible, since all consumers except of the lowest type $\theta_{1}$ will have an individual incentive to choose a contract devoted to a lower consumer type, as in the standard screening model with network effects. An incentive-compatible menu structure $\left\{\left(q_{i}, t_{i}\right)\right\}_{i=1}^{n}$ should satisfy participation constraints $\left(P_{i}\right)$ and the following set of incentive constraints:

$$
\theta_{i} V\left(q_{i}, \bar{q}\right)-t_{i} \geq \theta_{i} V\left(q_{j}, \bar{q}\right)-t_{j}
$$

for all $i, j \in N$, where $\bar{q}=\sum_{i \in N} q_{i} f\left(\theta_{i}\right)$ is the rationally expected equilibrium network size.

By adding incentive constraints $\left(I C_{i j}\right)$ and $\left(I C_{j i}\right)$, we see that

$$
\left(\theta_{i}-\theta_{j}\right)\left[V\left(q_{i}, \bar{q}\right)-V\left(q_{j}, \bar{q}\right)\right] \geq 0
$$

should hold for all $i, j \in N$. Since $V_{1}>0$, in order to have an implementable mechanism, the quantity scheme $q(\theta)$ should be a non-decreasing function of the type. We will refer to this condition as the monotonicity constraint.

The reason why we end up with exactly the same implementability conditions as in the standard screening problem without network effects is because in the incentive constraints we require only that no consumer has any incentive to deviate individually from his equilibrium choice. Since we have assumed a continuum of consumers in each type, a single consumer's choice cannot have a significant effect on network size, so $\bar{q}$ remains unchanged if other consumers stick to their equilibrium choice.

As standard in incentive theory literature, we first analyze the set of constraints to find the relevant ones. 
Lemma 1 In the second-best optimum there are $n$ binding constraints: $\left(P_{1}\right)$, the participation constraint of the lowest-type consumer, and $\left(I C_{i(i-1)}\right)$ for $i=2, \ldots, n$, the downward local incentive constraints. Furthermore, the optimal tariffs are

$$
\begin{aligned}
t_{1} & =\theta_{1} V\left(q_{1}, \bar{q}\right), \text { and } \\
t_{i} & =\theta_{i} V\left(q_{i}, \bar{q}\right)-\sum_{j=1}^{i-1} \Delta \theta_{j} V\left(q_{j}, \bar{q}\right), \text { for } i=2, \ldots, n,
\end{aligned}
$$

where $\Delta \theta_{j}=\theta_{j+1}-\theta_{j}$.

Proof. See Appendix A.

These tariff equations demonstrate the standard intuition of second-degree discrimination: the surplus of the lowest type consumers is fully grasped, while consumers of higher types should get an information rent of $\sum_{j=1}^{i-1} \Delta \theta_{j} V\left(q_{j}, \bar{q}\right)$ in order to satisfy incentive compatibility.

By substituting the optimal tariff functions into the profit function, it simplifies to the following form: ${ }^{20}$

$$
\begin{aligned}
\Pi^{S B} & =\theta_{1} V\left(q_{1}, \bar{q}\right) f\left(\theta_{1}\right)+\sum_{i=2}^{n}\left[\theta_{i} V\left(q_{i}, \bar{q}\right)-\sum_{j=1}^{i-1} \Delta \theta_{j} V\left(q_{j}, \bar{q}\right)\right] f\left(\theta_{i}\right)-c \bar{q}= \\
& =\sum_{i \in N}\left(\theta_{i}-\Delta \theta_{i} \frac{1-F\left(\theta_{i}\right)}{f\left(\theta_{i}\right)}\right) V\left(q_{i}, \bar{q}\right) f\left(\theta_{i}\right)-c \bar{q} .
\end{aligned}
$$

The function $\Pi^{S B}$ should be maximized in $q$, with respect to the monotonicity constraint (3) and $q \geq 0$. We ignore these constraints for the moment, and check at the end whether they are satisfied in equilibrium. Then the optimal allocation $q^{S B}$ is characterized by the following first-order conditions:

$$
\begin{gathered}
\theta_{i} V_{1}\left(q_{i}, \bar{q}\right)+\sum_{j \in N} \theta_{j} V_{2}\left(q_{j}, \bar{q}\right) f\left(\theta_{j}\right)-\Delta \theta_{i} \frac{1-F\left(\theta_{i}\right)}{f\left(\theta_{i}\right)} V_{1}\left(q_{i}, \bar{q}\right)- \\
-\sum_{j \in N} \Delta \theta_{j}\left[1-F\left(\theta_{j}\right)\right] V_{2}\left(q_{j}, \bar{q}\right)=c, \text { for all } i \in N .
\end{gathered}
$$

\footnotetext{
${ }^{20}$ This simplified form contains a non-defined type parameter, $\theta_{n+1}$ in $\Delta \theta_{n}$. However, it does not play any role, since it is multiplied by $1-F\left(\theta_{n}\right)=0$.
} 
The first term is the first-best individual effect, while the second is the first-best network effect. The third subtracted term will be called second-best individual effect, and this has exactly the same form as in the standard screening model without network effects. This effect equals zero for consumers of type $\theta_{n}$, so produces 'no distortion at the top', and is positive for all other types.

However, there is the final sum to be subtracted, which will be called secondbest network effect, and is strictly positive for all consumers. The presence of a second-best term in the optimum condition for the highest type consumers is due to the fact that now all types' information rent is affected by total network size $\bar{q}$ as well, which contains $q_{n}$, while in standard incentive theory the information rent of the $i$ th type depends only on $q_{i-1}$. Its emergence already foreshadows the result that in the presence of network effects the 'no distortion at the top' result will no longer hold.

Since the network effects are the same in all first-order conditions, combining two of them gives

$$
\left(\theta_{i}-\Delta \theta_{i} \frac{1-F\left(\theta_{i}\right)}{f\left(\theta_{i}\right)}\right) V_{1}\left(q_{i}, \bar{q}\right)=\left(\theta_{j}-\Delta \theta_{j} \frac{1-F\left(\theta_{j}\right)}{f\left(\theta_{j}\right)}\right) V_{1}\left(q_{j}, \bar{q}\right)
$$

for all $i, j \in N$. Now let us examine the implications of the omitted constraints.

First, $\theta_{i}-\Delta \theta_{i} \frac{1-F\left(\theta_{i}\right)}{f\left(\theta_{i}\right)}$ should be positive for all consumers supplied with a positive quantity, since $\theta_{n}-\Delta \theta_{n} \frac{1-F\left(\theta_{n}\right)}{f\left(\theta_{n}\right)}=\theta_{n}$ and $V_{1}$ are both positive. If $\theta_{i}-\Delta \theta_{i} \frac{1-F\left(\theta_{i}\right)}{f\left(\theta_{i}\right)} \leq 0$, the $i$ th type (and by the monotonicity constraint all lower types) will be shut down, so $q_{i}=0$. Note that no shut-down condition has the same form as in the classical screening literature without network effects.

Second, since $V_{11} \leq 0$, in order to satisfy the monotonicity constraint, $\theta_{i}-$ $\Delta \theta_{i} \frac{1-F\left(\theta_{i}\right)}{f\left(\theta_{i}\right)}>\theta_{j}-\Delta \theta_{j} \frac{1-F\left(\theta_{i}\right)}{f\left(\theta_{i}\right)}$ should be satisfied for $i>j$, if the monopoly wants to separate type- $i$ and type- $j$ consumers. If $\theta_{i}-\Delta \theta_{i} \frac{1-F\left(\theta_{i}\right)}{f\left(\theta_{i}\right)} \leq \theta_{j}-$ $\Delta \theta_{j} \frac{1-F\left(\theta_{i}\right)}{f\left(\theta_{i}\right)}$, the two types will be bunched, that is $q_{i}=q_{j}$. A possible sufficient condition to avoid bunching is that $\Delta \theta_{i}$ is the same for all $i$ and $F(\theta)$ satisfies the monotone hazard rate property: $\frac{d}{d \theta}\left(\frac{1-F(\theta)}{f(\theta)}\right) \leq 0$.

In the following discussion we assume that in the optimal mechanism at least two different types are served and discriminated. 


\section{Comparison of different outcomes}

We have derived the first-order conditions for the perfect (first-degree) and incentive (second-best) discrimination cases, which characterize the equilibrium quantities, and thereby the equilibrium tariffs in the respective regimes. We have seen that in the second-best case the presence of network effects distorts the first-best allocations for all consumers. In the next proposition we prove that this is a strict downward distortion for all consumers.

Instead of solving these equation systems and comparing directly the equilibrium allocations, we develop a parametrized functional form that encompasses both regimes as optimal solutions for different parameter values, and then use monotone comparative statics tools to show that the optimal solution is a strictly monotone function of this parameter. Note that the original Monotone Selection Theorem derived by Milgrom and Shannon (1994, Theorem 4') shows that the conditions under which the set of maximizers of a supermodular function is only non-decreasing in response to an exogenous parameter. By arguing from firstorder conditions, Edlin and Shannon (1998) extend this result by showing that under some conditions the maximizer should be strictly increasing in at least one dimension. Our next lemma builds on their result by identifying a sufficient condition so that the maximizer is strictly increasing in all dimensions.

Lemma 2 Let $Q=R^{n}$ and $T=R$, and let $f: Q \times T \rightarrow R$ be a continuously differentiable function, which is supermodular in $q$ on $Q$, and has strictly increasing differences in $(q, t)$ on $Q \times T$. Furthermore, suppose that $f(q, t)$ has increasing marginal returns for all choice variables, that is $\frac{\partial^{2} \Pi}{\partial q_{i} \partial t}>0$ for all $q_{i}$. Let $q(t) \in \underset{q \in Q}{\arg \max } \Pi(q, t)$. Then $q\left(t^{\prime \prime}\right)>q\left(t^{\prime}\right)$ if $t^{\prime \prime}>t^{\prime}$.

Proof. See Appendix B.

Armed with this lemma, we are able to give a simple proof for our main theorem. 
Proposition 3 The second-best allocation is strictly smaller than the first best allocation, that is $q^{S B}<q^{F B}$.

Proof. Consider only the types that are not shut down in the second-best regime (set $S$ ), since for the others the strict downward distortion holds trivially. Let us take the following parametrized form $\Pi: Q \times T \rightarrow R$ :

$$
\Pi(q, \alpha)=\sum_{i \in S}\left(\theta_{i}+\alpha \Delta \theta_{i} \frac{1-F\left(\theta_{i}\right)}{f\left(\theta_{i}\right)}\right) V\left(q_{i}, \bar{q}\right) f\left(\theta_{i}\right)-c \bar{q},
$$

where $T=[-1,0]$. When $\alpha=0$, we have the first-best profit function given in (1), while for $\alpha=-1$ we have the second-best profit function given in (4). Then the function $\Pi(q, \alpha)$ is supermodular in $q$ on $Q$ for all $\alpha$, since by the no shut-down condition the multipliers of $V(\cdot)$ are always positive for all $i \in S$. Moreover, $\Pi(q, \alpha)$ has strictly increasing differences in $(q, \alpha)$ on $Q \times T$, and the marginal returns of all $q_{i}$-s are increasing in $\alpha$, since $\frac{\partial^{2} \Pi}{\partial q_{i} \partial \alpha}>0$ for all $q_{i}$. Therefore, since $\alpha^{F B}>\alpha^{S B}$, Lemma 2 ensures that $q\left(\alpha^{F B}\right)>q\left(\alpha^{S B}\right)$.

Second, we compare the equilibrium allocations of first- and second-best discrimination regimes to the perfectly competitive case, where identical firms supply the network good at a price equal to marginal cost $c$. Then each consumer of type $\theta_{i}$ derives a utility of

$$
\theta_{i} V\left(q_{i}, \bar{q}^{P C}\right)-c q_{i}
$$

where $\bar{q}^{P C}$ is the expected network size under perfect competition, which should be fulfilled in equilibrium.

Maximizing utility in $q_{i}$ results in the first-order condition of

$$
\theta_{i} V_{1}\left(q_{i}, \bar{q}^{P C}\right)=c, \text { for all } i \in N \text {. }
$$

Let us denote the solution of this equation system by $q^{P C}$. By combining two first-order conditions, we see that $q_{i}^{P C}>q_{j}^{P C}$ if $\theta_{i}>\theta_{j}$. 
Proposition 4 The equilibrium allocation under perfect competition is strictly smaller than in the first-best discrimination case, that is $q^{P C}<q^{F B}$.

Proof. If we compare first-order conditions (6) with the first-order conditions (2) of the first-best discrimination case, we see that

$$
\theta_{i} V_{1}\left(q_{i}^{F B}, \bar{q}^{F B}\right)<\theta_{i} V_{1}\left(q_{i}^{P C}, \bar{q}^{P C}\right)
$$

for all $i \in N$. In the first-best case, the monopoly is supplying the welfaremaximizing allocation, so $\bar{q}^{F B}$ cannot be smaller than $\bar{q}^{P C}$, since the externalities are positive. Then since $V_{12} \geq 0$, for all $q_{i}^{F B}$

$$
V_{1}\left(q_{i}^{F B}, \bar{q}^{P C}\right) \leq V_{1}\left(q_{i}^{F B}, \bar{q}^{F B}\right)
$$

Combining this inequality with the former one, we have that

$$
\theta_{i} V_{1}\left(q_{i}^{F B}, \bar{q}^{P C}\right)<\theta_{i} V_{1}\left(q_{i}^{P C}, \bar{q}^{P C}\right),
$$

which yields that $q_{i}^{F B}>q_{i}^{P C}$ for all $i \in N$, since $V_{11} \leq 0$.

Thus, the perfectly competitive outcome is smaller than the first-best discrimination outcome. This is because perfectly competitive firms cannot internalize the network effects implied by larger allocations, since they cannot influence the quantity choice of the consumers. On the other hand, the perfectly discriminating monopoly can set the (larger) socially optimal allocation, and reap the increased surplus of each consumer.

The comparison of the allocations under second-best discrimination and perfect competition (defined by equations (5) and (6)) does not give unambiguous results, since we are comparing two outcomes, which fail to be the first-best for two different reasons: incentive problems due to information asymmetry and the incapability of internalizing network effects. However, if the impact of network effects is large enough to offset the effect due to the decrease in individual consumption (loosely speaking, if $V_{2}$ is sufficiently larger than $\left.\Delta \theta_{i} \frac{1-F\left(\theta_{i}\right)}{f\left(\theta_{i}\right)} V_{1}\right)$, then we may have a larger allocation in the screening monopoly regime than under perfect competition. 
Last, we show that the equilibrium outcome is a strictly increasing function of the intensity of the network effects, no matter which type of discrimination we consider. As a corollary, we can state that discrimination in the presence of network effects always leads to a larger allocation than in the standard screening case (i.e. without network effects). This result is natural in the case of first-best discrimination, but it also shows that despite the downward distorting factor from the first-best allocation, the presence of network effects has a positive impact in total on the resulting allocation in the second-best case as well.

Let us consider the utility function $U\left(\theta_{i}\right)=\theta_{i} V\left(q_{i}, \beta \bar{q}\right)-t_{i}$, where $\beta \geq 0$ measures the intensity of network size. If $\beta=0$, we are back to the standard discrimination case without positive network effects, while our original model refers to $\beta=1$.

Proposition 5 Both for first-and second-best discrimination in the presence of network effects, the equilibrium allocation $q(\beta)$ is a strictly increasing function of $\beta$ for the types supplied with positive quantities.

Proof. The set of types supplied with positive quantities is $N$ in the first-best regime and $S$ for the second-best regime, where $S$ is defined by the same no shut-down condition for any network size intensity. The profit function takes the form of $\Pi^{j}: Q \times T \rightarrow R$ :

$$
\Pi^{j}(q, \beta)=\sum\left(\theta_{i}+\alpha^{j} \Delta \theta_{i} \frac{1-F\left(\theta_{i}\right)}{f\left(\theta_{i}\right)}\right) V\left(q_{i}, \beta \bar{q}\right) f\left(\theta_{i}\right)-c \bar{q},
$$

where $\beta \in T=R_{+}$. The higher index $j$ shows whether we are in the first-or second-best regime, so $\alpha^{F B}=0, \alpha^{S B}=-1$. We have seen that the function $\Pi^{j}(q, \beta)$ is supermodular in $q$ on $Q$ for each $\beta$ and $j$. Now $\Pi^{j}(q, \beta)$ has strictly increasing differences in $(q, \beta)$ only on $Q \times(T \backslash\{0\})$, so applying Lemma 2 shows only that $q(\beta)$ is strictly increasing for $\beta \in(0, \infty)$. However, the Monotone Comparative Statics Theorem guarantees the smallest elements of $q(\beta)$ is increasing on the whole $T$, and if there exists a sufficiently small $\beta^{\prime}>0$ such that the smallest element of $q\left(\beta^{\prime}\right)$ equals the unique $q(0)$, then $q\left(\beta^{\prime} / 2\right)<q(0)$, which is a contradiction. Therefore $q(\beta)$ should be strictly increasing on $T$. 


\section{Discussion of the continuous-type case}

In the screening literature it is common to discuss the models in a continuoustype framework, since it simplifies considerably the exposition of the implementation problem. ${ }^{21}$ Indeed, the description of first- and second best optimal contracts presented in Section 3 and 4 can be easily modified to account for a continuum of types, and the characterizing conditions will have exactly the same form stated in integrals instead of sums. In this case, however, defining strategic complementarity and proving the supermodularity of the general profit function, which is the key for the monotone comparative statics results of Section 5 , is not straightforward. In this section we briefly sketch the analysis and the arising difficulties, which leads to slightly weaker results.

Suppose now that $\theta$ is distributed on $[\underline{\theta}, \bar{\theta}]$ according to a continuous distribution function $F(\theta)$, with a positive density $f(\theta)$ at each point. As in the discrete-type case, the relevant decision variables are the quantity choices of the monopoly, since the optimal tariff schedule $t(\theta)$ will be determined by the optimal quantity schedule $q(\theta)$. Now let $Q$ be the set of bounded, piecewise continuous functions $q(\theta)$ defined on $[\underline{\theta}, \bar{\theta}]$, and the join and meet of two elements $q^{\prime}(\theta)$ and $q^{\prime \prime}(\theta)$ are defined as the upper and lower envelope of the two functions: $q^{\prime}(\theta) \vee q^{\prime \prime}(\theta)=\max \left\{q^{\prime}(\theta), q^{\prime \prime}(\theta)\right\}$ and $q^{\prime}(\theta) \wedge q^{\prime \prime}(\theta)=\min \left\{q^{\prime}(\theta), q^{\prime \prime}(\theta)\right\}$. Then $Q$ is a lattice, since it always contains the join and meet of any two elements.

So far in our model we have been working with a discrete distribution of types, that is $F(\theta)$ was an $n$-step function where the interval of possible types was divided into $n$ partitions. Let us call this partitioning $\Omega_{n}$, and take finer and finer partitions by increasing $n$ towards infinity in such a way that $\Omega_{n}$ uniformly converges to $\Omega$ characterized by the continuous distribution function $F(\theta)$. We have seen that for any finite $n$, the $\Omega_{n}$ partitioning of the type space yielded a supermodular objective function $\Pi(q)$ in $q$ on $R^{n}$, and now we show that this property holds in the limit on the lattice $Q$ defined above.

\footnotetext{
${ }^{21}$ See for example Section 7.3 of Fudenberg and Tirole (1991) or Appendix 3.1 of Laffont and Martimort (2002).
} 
Lemma 6 If $\Pi(q)$ is supermodular in $q$ on $Q$ for all finite $\Omega_{n}$ partitioning of the type space, then $\Pi(q(\theta))$ will be supermodular in $q(\theta)$ on $Q$.

Proof. See Appendix C.

Given this property, we can use the Monotone Selection Theorem to show that $q^{S B}(\theta) \leq q^{F B}(\theta)$. Note that we can show only the downward distortion result, but not in the strict sense. The proof of Theorem 3 relies on the finite dimension of the choice space, and by using the result that network size should decrease under second-degree discrimination, we can only show that $q^{S B}(\theta)$ is strictly smaller than $q^{F B}(\theta)$ for a set of positive measure.

\section{Concluding remarks}

In this paper we have derived a general model to analyze the second-degree price discrimination problem of a monopoly selling a network good exhibiting strategic complementarities. By using the tools of monotone comparative statics, we were able to give a full characterization of screening contracts. We have seen that strategic complementarities and asymmetric information together lead to a strict downward distortion for all consumers, and the equilibrium outcome is an increasing function of the intensity of the network effects. Additionally, we have shown that a discriminating monopoly may supply larger quantities for all consumers than a perfectly competitive industry.

A crucial feature of our model was that the optimal contracts are designed such that it is individually not profitable for deviating from the truthtelling equilibrium. However, the natural question arises whether it could be advantageous for some consumers to form a coalition to coordinate their decisions and then reallocate the goods among themselves. Jeon and Menicucci (2002) show that there is no loss of generality in restricting our attention to contracts that satisfy only individually incentive compatibility constraints, if the coalitions are formed under asymmetric information. This is because buyers fail to realize the gains from joint deviations due to the transaction costs of asymmetric informa- 
tion among themselves, and the monopoly can use this fact to construct a menu of contracts, by which it can do at least as well as when there is no coalition. Although network effects are not present in their model, the intuition seems to hold in our setting as well.

In the whole paper the network goods provided to different types of consumers were assumed to be compatible with each other. Let us briefly discuss the case of two types where the monopoly chooses the network good provided to low-type consumers to be incompatible with the high-types' goods, while the high-types's good has full compatibility. Now low-types benefit less from the network, so the monopoly cannot charge such a high tariff for them. However, a high-type consumer will now have less incentive to choose the menu devoted to low-type consumers, since then he excludes himself from using a part of the network, so information rent of high-type consumers should decrease as well, which is profitable for the monopoly. Therefore, it is a natural conjecture that if the monopoly chooses to make its good partially incompatible, then it will choose to do so with the good devoted to low-type consumers, since high-type consumers have a higher marginal utility for the network.

If the good devoted to low-type consumers is incompatible with the good devoted to high-type consumers, then in equilibrium low-type consumers' utilities depend only on low-type consumers' choices. This is exactly the same case as if low-type consumers had the pessimistic expectation that high-type consumers will stay out of the market, so the monopoly has to design the contract devoted to low-type consumers such that they would accept it 'without the high-types' as well. But if high-type consumers observe the contract devoted to low-type consumers, no matter how pessimistic prior expectations they had about lowtypes' behavior, they will realize that low-types will accept that contract in any case. Then they will make their choices by expecting low-type ones 'in the network', thus the monopoly can design the menu devoted to high-types accordingly. This "divide-and-conquer" strategy, presented also in Jullien (2002) and Segal (2003), may help to overcome the problem of multiple equilibria induced by different consumers' expectations and to end up with unique implementation. 


\section{Appendices}

\section{A Proof of Lemma 1}

First, the participation constraint $\left(P_{i}\right)$ will be automatically satisfied for all types $i \geq 2$ if the constraints $\left(P_{1}\right)$ and $\left(I C_{i 1}\right)$ are satisfied, since

$$
\theta_{i} V\left(q_{i}, \bar{q}\right)-t_{i} \geq \theta_{i} V\left(q_{1}, \bar{q}\right)-t_{1}>\theta_{1} V\left(q_{1}, \bar{q}\right)-t_{1} \geq 0
$$

Second, by adding any two incentive constraints $\left(I C_{i j}\right)$ and $\left(I C_{j k}\right)$ such that $i>j>k$, we have

$$
\theta_{i} V\left(q_{i}, \bar{q}\right)-t_{i} \geq\left(\theta_{i}-\theta_{j}\right) V\left(q_{j}, \bar{q}\right)+\theta_{j} V\left(q_{k}, \bar{q}\right)-t_{k}
$$

Rearranging the monotonicity constraint (3) gives $\left(\theta_{i}-\theta_{j}\right) V\left(q_{j}, \bar{q}\right)+\theta_{j} V\left(q_{k}, \bar{q}\right) \geq$ $\theta_{i} V\left(q_{k}, \bar{q}\right)$. Therefore

$$
\theta_{i} V\left(q_{i}, \bar{q}\right)-t_{i} \geq \theta_{i} V\left(q_{k}, \bar{q}\right)-t_{k}
$$

so the incentive constraint $\left(I C_{i k}\right)$ is satisfied. The same reasoning can be done for the case of $i<j<k$, thus the local incentive constraints (the ones involving adjacent types) imply the global incentive constraints.

Now suppose that $\left(I C_{i j}\right)$ for $i<j$ will not be binding in equilibrium, so we ignore them for the moment and check later whether they will be satisfied. Then the remaining $n$ constraints constraints should be binding in equilibrium, implying the optimal tariff functions $\left(t_{1}\right)-\left(t_{n}\right)$. Finally, by substituting the corresponding tariff functions into the ignored upward local incentive constraints, we see that they are indeed satisfied if

$$
\Delta \theta_{i-1}\left[V\left(q_{i}, \bar{q}\right)-V\left(q_{i-1}, \bar{q}\right)\right] \geq 0, \text { for } i=2, \ldots, n,
$$

which is fulfilled by the monotonicity constraint (3). 


\section{B Proof of Lemma 2}

For any selections from this set of maximizers, the Monotone Selection Theorem ensures that $q\left(t^{\prime \prime}\right) \geq q\left(t^{\prime}\right)$ if $t^{\prime \prime}>t^{\prime}$, and from Theorem 3 of Edlin and Shannon (1998) we also know that $q\left(t^{\prime \prime}\right) \neq q\left(t^{\prime}\right)$, so they are different in at least one coordinates of $R^{n}$.

Now let us assume indirectly that $q\left(t^{\prime \prime}\right)$ is identical to $q\left(t^{\prime}\right)$ in $0<k<n$ coordinates, and they differ in the remaining $l=n-k$ coordinates. We may assume without any loss of generality that the matching coordinates are the first $k$ ones, so $q\left(t^{\prime \prime}\right)=\left(q_{k}^{\prime \prime}, q_{l}^{\prime \prime}\right)$ and $q\left(t^{\prime}\right)=\left(q_{k}^{\prime}, q_{l}^{\prime}\right)$, where $q_{k}^{\prime \prime}=q_{k}^{\prime}$, and $q_{l}^{\prime \prime}>q_{l}^{\prime}$. Now pin down the last $l$ coordinates to $q_{l}^{\prime}$ and find $q_{k}\left(q_{l}, t\right)=\arg \max f\left(q_{k}, q_{l}, t\right)$. Let $q_{k}\left(q_{l}^{\prime}, t^{\prime \prime}\right)$ be denoted by $q_{k}^{*}$. By definition $q_{k}\left(q_{l}^{\prime}, t^{\prime}\right)=q_{k}^{\prime}$, and by replicating the arguments given above, we see that $q_{k}^{*}$ cannot be smaller than $q_{k}^{\prime}$, and differs from $q_{k}^{\prime}$ in at least one coordinate. Based on these points, we can define $q^{*}=\left(q_{k}^{*}, q_{l}^{\prime}\right)$ and $q^{* *}=\left(q_{k}^{*}, q_{l}^{\prime \prime}\right)$, and then $q\left(t^{\prime \prime}\right) \vee q^{*}=q^{* *}$ and $q\left(t^{\prime \prime}\right) \wedge q^{*}=$ $q\left(t^{\prime}\right)$. Basically we are constructing a rectangle characterized by the points $q\left(t^{\prime}\right), q\left(t^{\prime \prime}\right), q^{*}$ and $q^{* *}$.

The supermodularity on $Q$ ensures that

$$
f\left(q\left(t^{\prime \prime}\right), t^{\prime \prime}\right)+f\left(q^{*}, t^{\prime \prime}\right) \leq f\left(q^{* *}, t^{\prime \prime}\right)+f\left(q\left(t^{\prime}\right), t^{\prime \prime}\right)
$$

and $f\left(q\left(t^{\prime}\right), t^{\prime \prime}\right)<f\left(q^{*}, t^{\prime \prime}\right)$ by the definition of $q^{*}$. These two conditions imply that $f\left(q^{* *}, t^{\prime \prime}\right)$ should be strictly larger than $f\left(q\left(t^{\prime \prime}\right), t^{\prime \prime}\right)$, which contradicts the indirect assumption that the optimal solution $q\left(t^{\prime \prime}\right)$ is identical to $q\left(t^{\prime}\right)$ in the first $k$ coordinates. This method can be applied for any positive $k$ (since the marginal returns of all $q_{i}$-s are increasing), so after $n-1$ steps we can conclude that $q\left(t^{\prime \prime}\right)$ should differ from $q\left(t^{\prime}\right)$ in all coordinates. 


\section{Proof of Lemma 6}

For any $\Omega_{n}$ partitioning, by the supermodularity of the function $\Pi(q)$ we have

$$
\Pi\left(q^{\prime}\right)+\Pi\left(q^{\prime \prime}\right) \leq \Pi\left(q^{\prime} \vee q^{\prime \prime}\right)+\Pi\left(q^{\prime} \wedge q^{\prime \prime}\right)
$$

for any $q^{\prime}, q^{\prime \prime} \in R^{n}$, where $q^{\prime} \vee q^{\prime \prime}=\left(\max \left\{q_{1}^{\prime}, q_{1}^{\prime \prime}\right\}, \ldots, \max \left\{q_{n}^{\prime}, q_{n}^{\prime \prime}\right\}\right)$ and $q^{\prime} \wedge q^{\prime \prime}=\left(\min \left\{q_{1}^{\prime}, q_{1}^{\prime \prime}\right\}, \ldots, \min \left\{q_{n}^{\prime}, q_{n}^{\prime \prime}\right\}\right)$. Note that each $n$-dimension vector $q$ can be represented by an $n$-step function $q_{n}(\theta)$, so the previous inequality can be reformulated in terms of step functions:

$$
\Pi\left(q_{n}^{\prime}(\theta)\right)+\Pi\left(q_{n}^{\prime \prime}(\theta)\right) \leq \Pi\left(q_{n}^{\prime}(\theta) \vee q_{n}^{\prime \prime}(\theta)\right)+\Pi\left(q_{n}^{\prime}(\theta) \wedge q_{n}^{\prime \prime}(\theta)\right)
$$

where $q_{n}^{\prime}(\theta) \vee q_{n}^{\prime \prime}(\theta)=\max \left\{q_{n}^{\prime}(\theta), q_{n}^{\prime \prime}(\theta)\right\}$ and $q_{n}^{\prime}(\theta) \wedge q_{n}^{\prime \prime}(\theta)=\min \left\{q_{n}^{\prime}(\theta), q_{n}^{\prime \prime}(\theta)\right\}$.

Suppose that as we are taking finer and finer partitions, $\lim _{n \rightarrow \infty} q_{n}^{\prime}(\theta)=q^{\prime}(\theta)$ and $\lim _{n \rightarrow \infty} q_{n}^{\prime \prime}(\theta)=q^{\prime \prime}(\theta)$. Then by the continuity of the profit function, $\lim _{n \rightarrow \infty} \Pi\left(q_{n}^{\prime}(\theta)\right)=\Pi\left(q^{\prime}(\theta)\right)$ and $\lim _{n \rightarrow \infty} \Pi\left(q_{n}^{\prime \prime}(\theta)\right)=\Pi\left(q^{\prime \prime}(\theta)\right)$.

Now for any $\epsilon>0$ there exists a high enough $n$ such that $\left|q_{n}^{\prime}(\theta)-q^{\prime}(\theta)\right| \leq \epsilon$ and $\left|q_{n}^{\prime \prime}(\theta)-q^{\prime \prime}(\theta)\right| \leq \epsilon$, that is $q^{\prime}(\theta)-\epsilon \leq q_{n}^{\prime} \leq q^{\prime}(\theta)+\epsilon$ and $q^{\prime \prime}(\theta)-\epsilon \leq q_{n}^{\prime \prime} \leq$ $q^{\prime \prime}(\theta)+\epsilon$ for each $\theta \in[\underline{\theta}, \bar{\theta}]$. Therefore,

$$
\max \left\{q^{\prime}(\theta), q^{\prime \prime}(\theta)\right\}-\epsilon \leq \max \left\{q_{n}^{\prime}(\theta), q_{n}^{\prime \prime}(\theta)\right\} \leq \max \left\{q^{\prime}(\theta), q^{\prime \prime}(\theta)\right\}+\epsilon,
$$

so $\left|\max \left\{q_{n}^{\prime}(\theta), q_{n}^{\prime \prime}(\theta)\right\}-\max \left\{q^{\prime}(\theta), q^{\prime \prime}(\theta)\right\}\right| \leq \epsilon$ for each $\theta \in[\underline{\theta}, \bar{\theta}]$. The similar argument can be replicated for the minimum operator, so we have that the functional limit of the upper (lower) envelope of the two functions is the upper (lower) envelope of the functional limits of the two functions.

Then by the continuity of $\Pi(\cdot), \lim _{n \rightarrow \infty} \Pi\left(q_{n}^{\prime}(\theta) \vee q_{n}^{\prime \prime}(\theta)\right)=\Pi\left(q^{\prime}(\theta) \vee q^{\prime \prime}(\theta)\right)$ and $\lim _{n \rightarrow \infty} \Pi\left(q_{n}^{\prime}(\theta) \wedge q_{n}^{\prime \prime}(\theta)\right)=\Pi\left(q^{\prime}(\theta) \wedge q^{\prime \prime}(\theta)\right)$. Therefore, by taking the limits of inequality (7), we have

$$
\Pi\left(q^{\prime}(\theta)\right)+\Pi\left(q^{\prime \prime}(\theta)\right) \leq \Pi\left(q^{\prime}(\theta) \vee q^{\prime \prime}(\theta)\right)+\Pi\left(q^{\prime}(\theta) \wedge q^{\prime \prime}(\theta)\right) .
$$




\section{References}

[1] Cooper, R., and John, A. (1988), Coordinating coordination failures in Keynesian models, Quarterly Journal of Economics 103, 441-463.

[2] Edlin, A., and Shannon, C. (1998), Strict Monotonicity in Comparative Statistics, Journal of Economic Theory 81, 201-219.

[3] Ellison, G. and Fudenberg, D. (2000), The Neo-Luddite's Lament: Too Many Upgrades in the Software Industry, Rand Journal of Economics 31, 253-272.

[4] Farrel, J. and Saloner, G. (1985), Standardization, Compatibility, and Innovation, Rand Journal of Economics 16, 70-83.

[5] Fudenberg, D., and Tirole, J. (1991), Game Theory, MIT Press.

[6] Fudenberg, D. and Tirole, J. (2000), Pricing a Network Good to Deter Entry, Journal of Industrial Economics 48, 373-390.

[7] Hahn, J-H. (2003), Nonlinear Pricing of Telecommunications with Call and Network Externalities, International Journal of Industrial Organization 21, 949-967.

[8] Jeon, D-S. and Menicucci D. (2002), Buyer Coalition Against Monopolistic Screening: On the Role of Asymmetric Information among Buyers, working paper, Universitat Pompeu Fabra.

[9] Jullien, B. (2000), Participation Constraints in Adverse Selection Problems, Journal of Economic Theory 93, 1-47.

[10] Jullien, B. (2002), Competing in Network Industries: Divide and Conquer, working paper, IDEI, University of Toulouse.

[11] Katz, M. and Shapiro, C. (1985), Network Externalities, Competition, and Compatibility, American Economic Review 75, 424-440.

[12] Laffont, J-J. (1988), Fundamentals of public economics, MIT Press. 
[13] Laffont, J-J., and Martimort, D. (2002), The Theory of Incentives: The Principal-Agent Model, Princeton University Press.

[14] Liebowitz, S. and Margolis, S. (2002), Network Effects, in Cave, M. et al. (editors), Handbook of Telecommunications Economics, Volume 1, 75-96, Elsevier Science.

[15] Littlechild, S. (1975), Two-Part Tariffs and Consumption Externalities, Bell Journal of Economics 6, 661-670.

[16] Lockwood, B. (2000), Production Externalities and Two-way Distortion in Principal-multi-agent Problems, Journal of Economic Theory 92, 144-166.

[17] Maskin, E. and Riley, J. (1984), Monopoly with Incomplete Information, Rand Journal of Economics 15, 171-196.

[18] Milgrom, P., and Roberts, J. (1990), Rationalizability, Learning, and Equilibrium in Games with Strategic Complementarities, Econometrica 58, 1255-1277.

[19] Milgrom, P., and Roberts, J. (1994), Comparing Equilibria, American Economic Review 84, 441-459.

[20] Milgrom, P. and Roberts, J.(1996), Coalition-Proofness and Correlation with Arbitrary Communication Possibilities, Games and Economic Behavior 17, 113-28.

[21] Milgrom, P., and Shannon, C. (1994), Monotone Comparative Statistics, Econometrica 62, 157-180.

[22] Mussa, M. and Rosen, S., (1978), Monopoly and Product Quality, Journal of Economic Theory 18, 301-317.

[23] Oren, S., Smith, S. and Wilson, R. (1982), Nonlinear Pricing in Markets with Interdependent Demand, Marketing Science 1, 287-313. 
[24] Rohlfs, J. (1974), A Theory of Interdependent Demand for a Communication Services, Bell Journal of Economics 5, 16-37.

[25] Segal, I., (1999), Contracting with Externalities, Quarterly Journal of Economics 114, 337-388.

[26] Segal, I. (2003), Coordination and Discrimination in Contracting with Externalities: Divide and Conquer?, Journal of Economic Theory 113, 147181.

[27] Sharkey (1982), The Theory of Natural Monopoly, Cambridge University Press.

[28] Topkis, D. (1978), Minimizing a Submodular Function on a Lattice, Operations Research 26, 305-321.

[29] Topkis, D. (1998), Supermodularity and Complementarity, Princeton University Press. 